\title{
Dengue outlook for the World Cup in Brazil: an early warning model framework driven by real-time seasonal climate forecasts
}

Rachel Lowe, Christovam Barcellos, Caio A S Coelho, Trevor C Bailey, Giovanini Evelim Coelho, Richard Graham, Tim Jupp, Walter Massa Ramalho, Marilia Sá Carvalho, David B Stephenson, Xavier Rodó

\section{Summary}

Background With more than a million spectators expected to travel among 12 different cities in Brazil during the football World Cup, June 12-July 13, 2014, the risk of the mosquito-transmitted disease dengue fever is a concern. We addressed the potential for a dengue epidemic during the tournament, using a probabilistic forecast of dengue risk for the 553 microregions of Brazil, with risk level warnings for the 12 cities where matches will be played.

Methods We obtained real-time seasonal climate forecasts from several international sources (European Centre for Medium-Range Weather Forecasts [ECMWF], Met Office, Meteo-France and Centro de Previsão de Tempo e Estudos Climáticos [CPTEC]) and the observed dengue epidemiological situation in Brazil at the forecast issue date as provided by the Ministry of Health. Using this information we devised a spatiotemporal hierarchical Bayesian modelling framework that enabled dengue warnings to be made 3 months ahead. By assessing the past performance of the forecasting system using observed dengue incidence rates for June, 2000-2013, we identified optimum trigger alert thresholds for scenarios of medium-risk and high-risk of dengue.

Findings Our forecasts for June, 2014, showed that dengue risk was likely to be low in the host cities Brasília, Cuiabá Curitiba, Porto Alegre, and São Paulo. The risk was medium in Rio de Janeiro, Belo Horizonte, Salvador, and Manaus. High-risk alerts were triggered for the northeastern cities of Recife $\left(p_{\text {high }}=19 \%\right)$, Fortaleza $\left(p_{\text {high }}=46 \%\right)$, and Natal $\left(\mathrm{p}_{\text {high }}=48 \%\right)$. For these high-risk areas, particularly Natal, the forecasting system did well for previous years (in June, 2000-13).

Interpretation This timely dengue early warning permits the Ministry of Health and local authorities to implement appropriate, city-specific mitigation and control actions ahead of the World Cup.

Funding European Commission's Seventh Framework Research Programme projects DENFREE, EUPORIAS, and SPECS; Conselho Nacional de Desenvolvimento Científico e Tecnológico and Fundação de Amparo à Pesquisa do Estado do Rio de Janeiro.

\section{Introduction}

Dengue is an emerging vector-borne disease. Half of the world's population live in dengue endemic regions, particularly in southeast Asia, the Pacific region, and the Americas. ${ }^{1}$ About $80 \%$ of people infected with dengue virus are asymptomatic. Approximately $5 \%$ of people diagnosed with dengue have more severe illness and $1 \%$ have severe life-threatening infections. ${ }^{2}$ Its recent expansion has been attributed to a combination of urbanisation, poor living conditions, international global travel and trade, changes in mosquito distribution and abundance, climate variability, and climate change. ${ }^{3,4}$ Epidemic dengue transmission has a seasonal pattern, because of the influence of temperature and rainfall on mosquito abundance and capacity, ${ }^{5}$ with increased incidence at the end of the summer, in the rainy season, and in warm periods. ${ }^{6}$

The interaction between climate and dengue transmission dynamics can vary between different ecological zones. ' Local living conditions, such as demographic density, population mobility, mosquito infestation, and sanitation are important collective risk factors. ${ }^{8}$ Poor sanitation conditions such as inadequate refuse collection services and water supply encourage mosquito breeding sites. Climatic factors interact with local conditions, affecting mosquito infestation, human susceptibility, and the contact rate between both populations. $^{8}$

Transmission of all four dengue virus serotypes occurs via a mosquito-human cycle. ${ }^{9}$ After an incubation period of 7-14 days, vectors (Aedes aegypti deemed the main vector) become infectious and can transmit the virus by biting human hosts. The human incubation period is typically 4-7 days. For transmission to mosquitoes, they must feed on an infected person during the 5 days when large amounts of virus are in the blood. Overall, 3 weeks are required for the virus to pass between two human hosts. Epidemics depend on large numbers of mosquitoes, a susceptible human population, and high rate of contact between mosquitoes and humans. The vector lifecycle and vectorial capacity is related to temperature, ${ }^{9,10}$ whereas
Lancet Infect Dis 2014; 14: 619-26

Published Online

May 17, 2014

http://dx.doi.org/10.1016/ S1473-3099(14)70781-9

See Comment 543

Climate Dynamics and Impacts Unit, Institut Català de Ciències del Clima (IC3), Barcelona, Spain (R Lowe PhD, Prof X Rodó PhD); Fundação Oswaldo Cruz (FIOCRUZ), Rio de Janeiro, RJ, Brazil (C Barcellos PhD,

M Sá Carvalho PhD); Centro de Previsão de Tempo e Estudos Climáticos, Instituto Nacional de Pesquisas Espaciais, Cachoeira Paulista, SP, Brazil (CA S Coelho PhD); Exeter Climate Systems, College of Engineering, Mathematics and Physical Sciences, University of Exeter, Exeter, UK (Prof T C Bailey PhD, T Jupp PhD, Prof D B Stephenson PhD); Coordenação Geral do Programa Nacional de Controle da Dengue, Ministério da Saúde, Brasília, DF, Brazil (G E Coelho PhD); Met Office Hadley Centre, Exeter, Devon, UK (R Graham OBE PhD); Faculdade de Ceilândia, Universidade de Brasília, Brasília, DF, Brazil (W Massa Ramalho MSc); and Institució Catalana de Recerca i Estudis Avançats, Barcelona, Spain (Prof X Rodó)

Correspondence to: Dr Rachel Lowe, Climate Dynamics and Impacts Unit, Institut Català de Ciències del Clima (IC3), Doctor Trueta 203, 08005 Barcelona, Spain rachel.lowe@ic3.cat 
the reproduction rate can depend, for example, on rainfall to fill discarded containers outdoors to create mosquito breeding sites. Human transmission is enhanced by dense urban populations and controlled by human serotype-specific herd immunity.

This century, Brazil has reported more cases of dengue fever than anywhere else in the world, ${ }^{11}$ with more than 7 million up to 2013. Many cities have climate conditions conducive to the proliferation and vectorial capacity of A aegypti. Brazil will soon host the 2014 Fédération Internationale de Football Association (FIFA) World Cup, an international men's football tournament. More than a million spectators are expected to travel between 12 different cities during June and July (the southern-hemisphere winter period). ${ }^{12}$

A news article by Simon Hay, ${ }^{13}$ with estimates based solely on averages of past dengue cases, stimulated a debate about the risk of dengue fever in Brazil during the World Cup. The possibility of a large dengue fever outbreak during the World Cup, capable of infecting visitors and spreading dengue back to their home destinations depends on the combination of many factors, such as mosquito density and the population susceptibility in the host country. ${ }^{14}$

Several preventive measures are routine in cities hosting the World Cup, both for endemic and epidemic situations: entomological surveillance and vector control measures, notification of suspect cases with laboratory confirmation, and clinical care protocols. ${ }^{15}$ Usually, epidemic detection is based on identification of the circulating dengue virus serotypes and the number of notified suspected cases. In response to an increase in the number of cases, especially severe clinical presentations, media campaigns are launched and health services prepare to increase access and adequate treatment. ${ }^{16}$ However, by this time, it is usually too late to decrease the infection rate. No alarm trigger exists for the third element of the transmission chain, the mosquito. All large cities are infested with aedes and methods to detect increases in mosquito populations are not well established. Further, under favourable conditions for feeding and reproduction, the increase of the aedes population might be exponential. ${ }^{17}$ Both mosquito density and susceptible populations vary substantially between the host cities located in ecologically diverse zones.

Complex transmission dynamics, interplay of environmental and social factors, and the interaction of different dengue serotypes make the construction and use of predictive models a challenge, involving different temporal and spatial scales. Despite these challenges, dengue modelling provides an important basis for early warning systems for improved control. Due to time lags implicated in the climate dengue transmission system, which are typically reported to be 1-3 months, ${ }^{6,10}$ observed climate variables can provide crucial predictive lead capacity for forecasting dengue epidemics. This lead time can be extended by using climate forecasts. To date, the incorporation of real-time climate forecasts into public health decision systems has been rare.

We address the potential for a dengue epidemic during the tournament, by providing probabilistic forecasts of dengue risk for the 553 microregions of Brazil with risk-level warnings issued for the 12 cities where the matches will be played. The dengue early warning system, formulated using a Bayesian spatiotemporal model framework, ${ }^{18,19}$ is driven by realtime seasonal climate forecasts ${ }^{20}$ and the epidemiological situation in Brazil at the forecast issue date. Seasonal climate forecasts have been reported to have the most skill (a statistical assessment of the quality of forecasts when compared with observations) in tropical regions of Brazil, with moderate skill in extratropical regions. ${ }^{20}$

\section{Methods \\ Data}

We obtained dengue data from the Notifiable Diseases Information System (SINAN), organised by the Brazilian Ministry of Health and available via the Health Information Department (DATASUS). We included in the analysis confirmed cases of dengue fever, including mild infections, dengue haemorrhagic fever, and shock syndrome, from 2000 to 2013, and we summarised them by month and microregion. A microregion typically consists of one large city and several smaller municipalities. The mean microregion area is $14200 \mathrm{~km}^{2}$, ranging from $17000 \mathrm{~km}^{2}$ to $332000 \mathrm{~km}^{2}$. Cases are routinely confirmed by clinical and epidemiological evidence. The quality of the dengue dataset depends on the technical and operational system of epidemiological surveillance in every geographic area to detect, report, investigate, and do specific laboratory tests to confirm the diagnosis of dengue cases. These activities are done by health surveillance services at the municipal level, under supervision of a national coordinating service. Under-reporting might result from non-declared or self-diagnosed cases or be attributed to difficulties in the identification of the clinical forms, mainly for the mild and moderate manifestations of infections. Overestimation occurs during some epidemics because of public and health-service awareness. As such, the dataset will contain errors for the exact magnitude and timing of epidemics. These factors underestimate incidence during the interepidemic periods and overestimate incidence during epidemic periods. However, since the goal of this study was to detect outbreaks, by predicting the probability of exceeding epidemic dengue thresholds (rather than deterministic incidence rates) the effect of misreporting on model results was minimised.

The Ministry of Health made case data available for February, 2014, to make the dengue forecast for June, 2014. We derived social and environmental variables, such as demographic density, urban population, and biome 


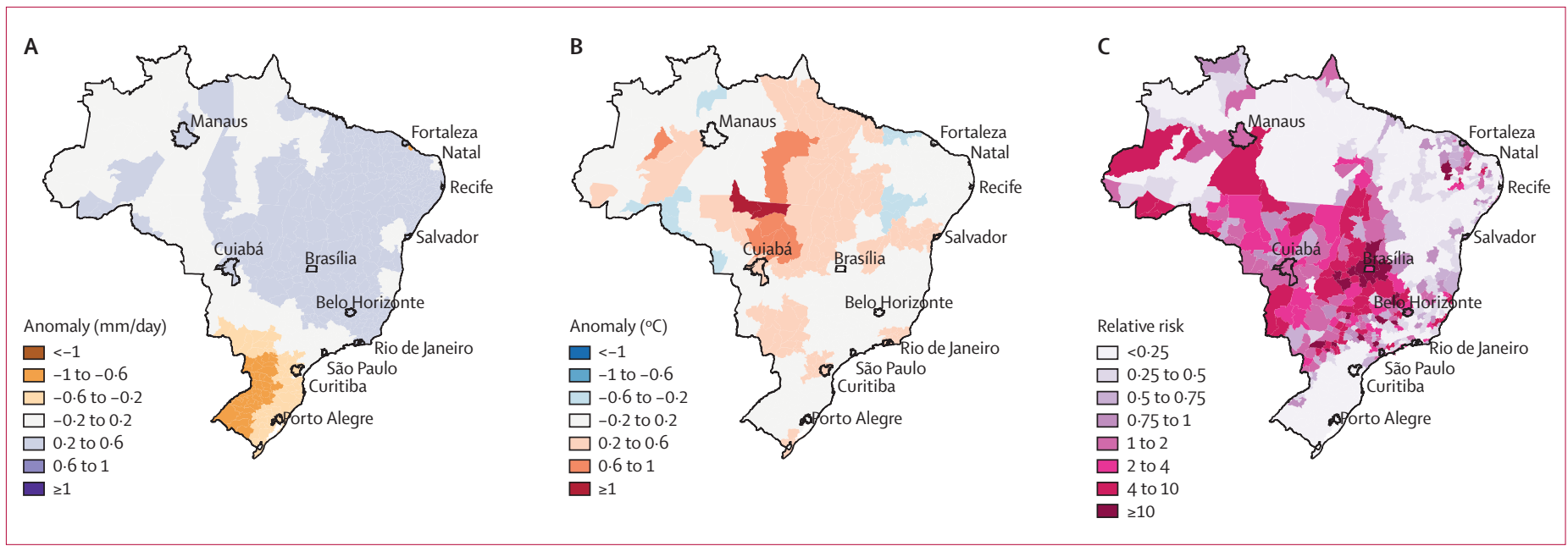

Figure 1: Dengue forecast drivers

(A) Precipitation ( $\mathrm{mm}$ per day) and (B) temperature $\left({ }^{\circ} \mathrm{C}\right.$ ) anomaly (departure from the long-term average) forecasts March-May, 2014, produced in February, 2014 by the Center for Weather Forecasting and Climate Research (CPTEC). (C) Observed dengue relative risk (observed-to-expected cases), February, 2014, collated in March, 2014, by the Brazilian Ministry of Health.

categories, from the 2000 and 2010 demographic census, which was available from the Brazilian Climate and Health Observatory. We estimated population values by the exponential interpolation of census data.

We extracted monthly precipitation and temperature anomalies from 1981 to 2013 (spatial resolution of $2.5^{\circ} \times 2 \cdot 5^{\circ}$ latitude-longitude grids, where a grid square over Brazil has an approximate area of $75625 \mathrm{~km}^{2}$, $1^{\circ} \approx 110 \mathrm{~km}$ ), from the Global Precipitation Climatology Project (GPCP) version 2.2 Combined Precipitation Data Set $^{21}$ and Climate Prediction Center (CPC) Global Historical Climatology Network (GHCN)/ Climate Anomaly Monitoring System (CAMS) Monthly Global Surface Air Temperature Data Set. ${ }^{22}$ We produced the temperature forecast using an empirical model previously described. ${ }^{20}$ This empirical model uses previous January Pacific and Atlantic sea surface temperatures as predictor variables for March, April, and May temperatures over Brazil. The precipitation forecast for March, April, and May, issued in February, is from the EUROBRISA integrated system. ${ }^{20}$ Briefly, the precipitation forecasts are generated using a combination of three dynamical (climate model based) prediction systems and one statistical or empirical system. The dynamical systems used are those of European Centre for Medium-Range Weather Forecasts (ECMWF; System 4), the Met Office (Global Seasonal Prediction System, version 5 [GloSea 5]), and Meteo-France (System 4). The individual precipitation forecasts are combined and calibrated using a Bayesian approach, known as forecast assimilation, ${ }^{23}$ to produce the EUROBRISA integrated forecast used here in this study.

We collated the multisourced spatiotemporal datasets and we reconciled gridded climate data and microregion level data (ie, dengue, demographic, and cartographic data) by assigning a grid point to each microregion on

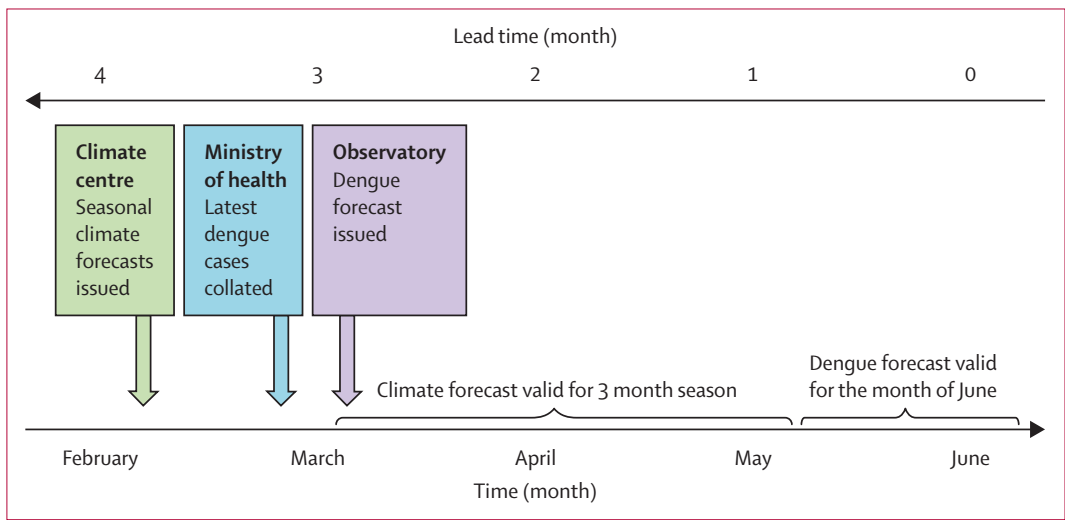

Figure 2: Dengue forecast lead-time schematic

Schematic to show lead-time gained from using the combined and calibrated multimodel 3 month average (March, April, May) precipitation forecasts from the EUROBRISA integrated system and temperature forecasts produced with the empirical model described in (Coelho et $\mathrm{al}^{20}$ ), produced in mid-February by the Center for Weather Forecasting and Climate Research (CPTEC) and the latest dengue cases from the Ministry of Health, Brazil (February estimate collated during March). The probabilistic dengue forecast, driven by climate forecasts and current dengue risk, could be issued by the climate and health observatory by mid-March. This provides a forecast lead-time of 3 months.

the basis of the shortest Euclidean distance between the microregion centroid and neighbouring grid points.

For the Brazilian Climate and Health Observatory see http:// www.climasaude.icict.fiocruz.br

\section{Dengue model formulation and forecasts}

We formulated a spatiotemporal hierarchical Bayesian For more on EUROBRISA see model $^{18,19}$ to model monthly dengue cases, from 2000 to 2013, for 553 Brazilian microregions. Explanatory variables included population density, altitude, precipitation, and temperature (averaged over the preceding 3 months) and dengue relative risk lagged by 4 months. We included this relative risk in the model framework as the log ratio of observed-to-expected dengue cases (dengue relative risk) 4 months ahead for every microregion. We chose this lag as a compromise between the longest lag plausible to provide predictive 


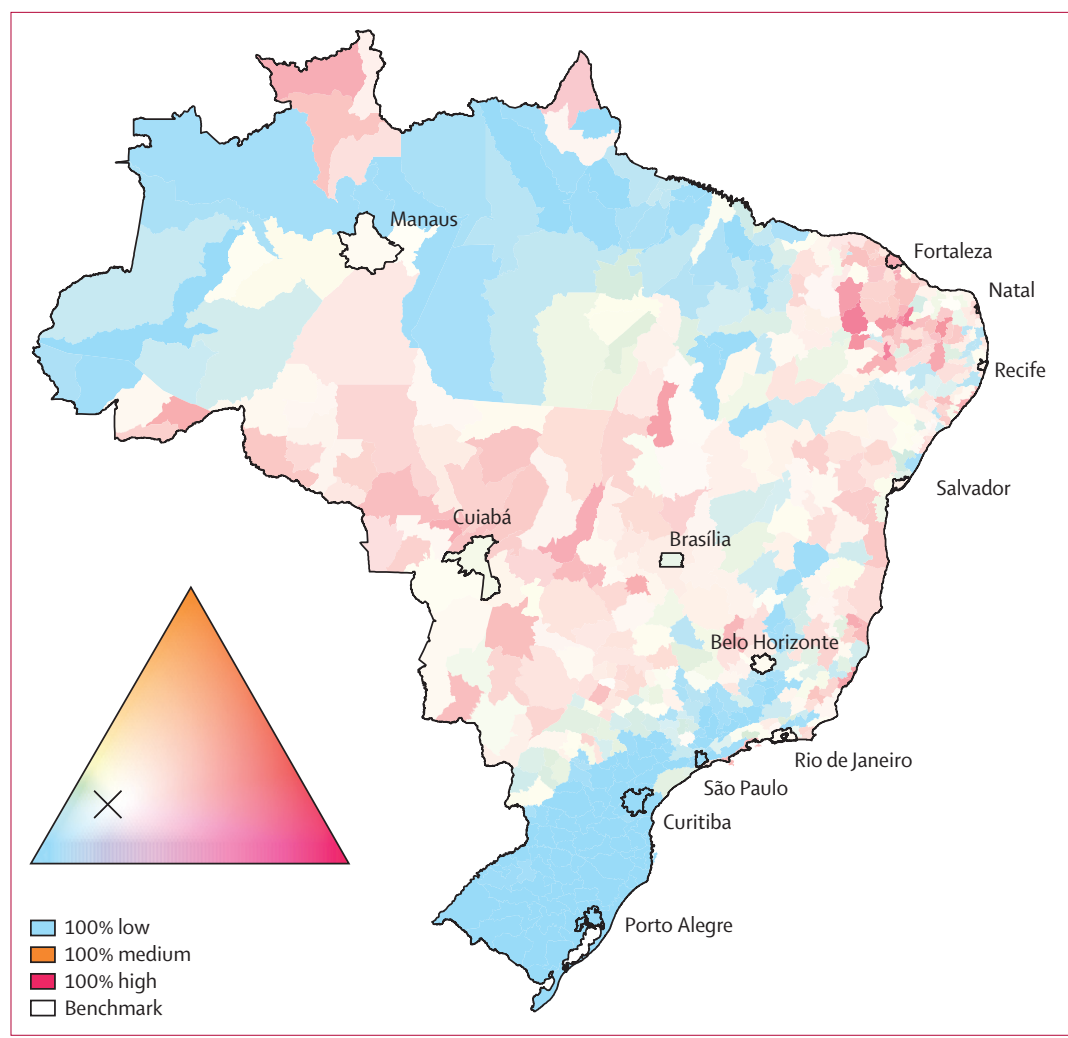

Figure 3: Probabilistic dengue forecast for Brazil, June, 2014

Dengue forecast for June, 2014. The continuous colour palette (ternary phase diagram) conveys the probabilities assigned to low-risk, medium-risk, and high-risk dengue categories. Category boundaries defined as 100 cases per 100000 inhabitants and 300 cases per 100000 inhabitants. The greater the colour saturation, the more certain is the forecast of a particular outcome. Strong red shows a high probability of high dengue risk. Strong blue indicates a high probability of low dengue risk. Colours close to white indicate a forecast similar to the benchmark (long-term average distribution of dengue incidence in Brazil, June, 2000-13: $\mathrm{p}_{\text {low }}=68 \%, \mathrm{p}_{\text {medium }}=16 \%$, $\mathrm{p}_{\text {high }}=16 \%$, marked by a cross.

See Online for appendix effects (appendix).

To produce the forecast for June, 2014, the model was driven with real-time seasonal precipitation and temperature forecasts (March-May, 2014), produced in February, 2014, and the observed epidemiological situation for February, 2014, collated in March, 2014 (figures 1 and 2). Posterior predictive distributions ${ }^{24}$ were simulated for every microregion to determine the probability of dengue incidence rates exceeding predefined risk thresholds.

To present the forecast to decision makers, we calculated the probability of dengue incidence falling into predefined categories. The Brazilian Ministry of Health is interested in areas in which dengue incidence in any given month is lower than 100 cases per 100000 inhabitants (low risk), between 100 and 300 cases per 100000 inhabitants (medium risk), and

\begin{tabular}{|c|c|c|c|c|c|}
\hline & \multirow[t]{2}{*}{ Warning } & \multicolumn{3}{|c|}{ Probability } & \multirow{2}{*}{$\begin{array}{l}\text { Skill score } \\
\text { (RPSS) }\end{array}$} \\
\hline & & $\mathrm{p}_{\text {low }}$ & $\mathrm{p}_{\text {medium }}$ & $\mathrm{P}_{\text {high }}$ & \\
\hline Belo Horizonte & Medium & $65 \%$ & $24 \%$ & $11 \%$ & 0.14 \\
\hline Brasília & Low & $73 \%$ & $20 \%$ & $7 \%$ & 0.14 \\
\hline Cuiabá & Low & $71 \%$ & $22 \%$ & $7 \%$ & 0.01 \\
\hline Curitiba & Low & $100 \%$ & 0 & 0 & 1 \\
\hline Fortaleza & High & $34 \%$ & $20 \%$ & $46 \%$ & 0.5 \\
\hline Manaus & Medium & $63 \%$ & $25 \%$ & $12 \%$ & 0.15 \\
\hline Natal & High & $32 \%$ & $20 \%$ & $48 \%$ & 0.67 \\
\hline Porto Alegre & Low & $100 \%$ & 0 & 0 & 1 \\
\hline Recife & High & $57 \%$ & $24 \%$ & $19 \%$ & 0.23 \\
\hline Salvador & Medium & $56 \%$ & $27 \%$ & $17 \%$ & 0.14 \\
\hline São Paulo & Low & $99 \%$ & $1 \%$ & 0 & 0.99 \\
\hline Rio de Janeiro & Medium & $62 \%$ & $25 \%$ & $13 \%$ & 0.21 \\
\hline \multicolumn{6}{|c|}{$\begin{array}{l}\text { RPPS=ranked probability skill score. Dengue risk warnings for June, 2014, for the } \\
\text { microregions in which the World Cup stadiums are located. Low risk was defined as } \\
\text { fewer than } 100 \text { cases per } 100000 \text { inhabitants, medium risk as between } 100 \text { and } \\
300 \text { cases per } 100000 \text { inhabitants, and high risk as greater than } 300 \text { cases per } \\
100000 \text { inhabitants. If the probability of low risk is less than } 68 \% \text {, a medium risk } \\
\text { warning is issued. If the probability of high risk is concurrently greater than } 18 \% \text {, the } \\
\text { warning is upgraded to high risk. The skill score (RPSS) indicates the past performance } \\
\text { forecasting system, with RPSS=1 showing a perfect forecasting system. }\end{array}$} \\
\hline \multicolumn{6}{|c|}{$\begin{array}{l}\text { Table: Dengue risk warnings for June, } 2014 \text { for stadium locations, } \\
\text { by microregion }\end{array}$} \\
\hline
\end{tabular}

higher than 300 cases per 100000 inhabitants (high risk). We used a novel visualisation technique ${ }^{25}$ to produce a map in which the forecast at every microregion is expressed as a colour determined by a combination of three probabilities (ie, probability of low, medium, and high risk; appendix).

\section{Model assessment}

To assess complex Bayesian models, posterior predictive distributions can be compared with observed data. ${ }^{24} \mathrm{We}$ obtained the posterior predictive distribution of the response variable (dengue cases) by simulating new pseudo-observations, using samples from the posterior distribution of the variables in the model, in crossvalidation mode (appendix). In other words, we refitted the model 14 times, leaving out 1 year at a time, to produce out-of-sample posterior predictive distributions (retrospective dengue forecasts), driven by corresponding past seasonal climate forecasts (March-May) and dengue relative risk (February) for the years 2000-13. We then compared predictions with observed data for June, 2000-13. We calculated the rank probability skill score (RPSS), ${ }^{26}$ which expresses forecast skill relative to the skill of a benchmark forecast for each microregion (ie, a forecast indicating $68 \%$ probability of low risk, $16 \%$ probability of medium risk, and $16 \%$ probability of high risk, based on the observed distribution of dengue incidence in Brazil, in June, 2000-13). The RPSS takes the value 1 for a perfect forecast and 0 if the forecast is no better than the benchmark reference (appendix). 


\section{Triggering risk alerts}

To determine optimum decision trigger thresholds for medium and high risk alerts, we calculated relative (or receiver) operating characteristic (ROC) curves for the binary events of exceeding epidemic thresholds of 100 and 300 cases per 100000 inhabitants. The ROC is a graph of hit rate (proportion of epidemics correctly predicted, or sensitivity) against false alarm rate (proportion of epidemics predicted but did not occur, or 1-specificity) for varying decision trigger thresholds. ${ }^{26} \mathrm{We}$ calculated the curves using the cross-validated past predictions compared with observed outcomes. We defined medium-risk $(\sigma)$ and high-risk $(\rho)$ trigger thresholds as the point on the curve closest to the point of perfect discrimination $(0,1)$. We then assigned warning levels to the microregions in which the World Cup stadiums are located. If the probability of low risk falls below $(1-\sigma)$, where $\sigma$ is the optimum medium risk alert trigger threshold, a medium alert is issued. If the probability of high risk concurrently exceeds $\rho$, the optimum high trigger alert threshold, the alert is upgraded to high risk.

\section{Role of the funding source}

The funders of the study had no role in study design, data collection, data analysis, data interpretation, or writing of the report. The corresponding author had full access to all the data in the study and had final responsibility for the decision to submit for publication.

\section{Results}

For the ternary probabilistic dengue forecasts for June, 2014, we defined risk category boundaries as 100 cases per 100000 inhabitants and 300 cases per 100000 inhabitants, the standard adopted by the Brazilian Ministry of Health (figure 3; appendix). In south Brazil and parts of the Amazon rainforest, the probability of low risk of dengue was strong, including the World Cup stadium cities of Porto Alegre, Curitiba, and São Paulo. Forecast probabilities also substantially favour low risk in Cuiabá and Brasília, located in the central region, although medium risk in June, 2014, was possible. We noted pockets of high risk in northeastern Brazil, with dengue incidence more likely to be high in the cities of Fortaleza and Natal. Meanwhile, the cities of Rio de Janeiro, Belo Horizonte, Salvador, and Manaus were forecast to have medium dengue risk levels (table).

We assessed the past performance skill of the forecasting system (figure 4) by calculating the RPSS for each microregion (appendix). Values greater than zero indicate the model provides more information beyond using the benchmark (long-term average distribution of dengue incidence, June, 2000-13). The closer the skill score to one, the more likely the model will correctly predict risk categories. Negative values indicate that the model performs worse than the

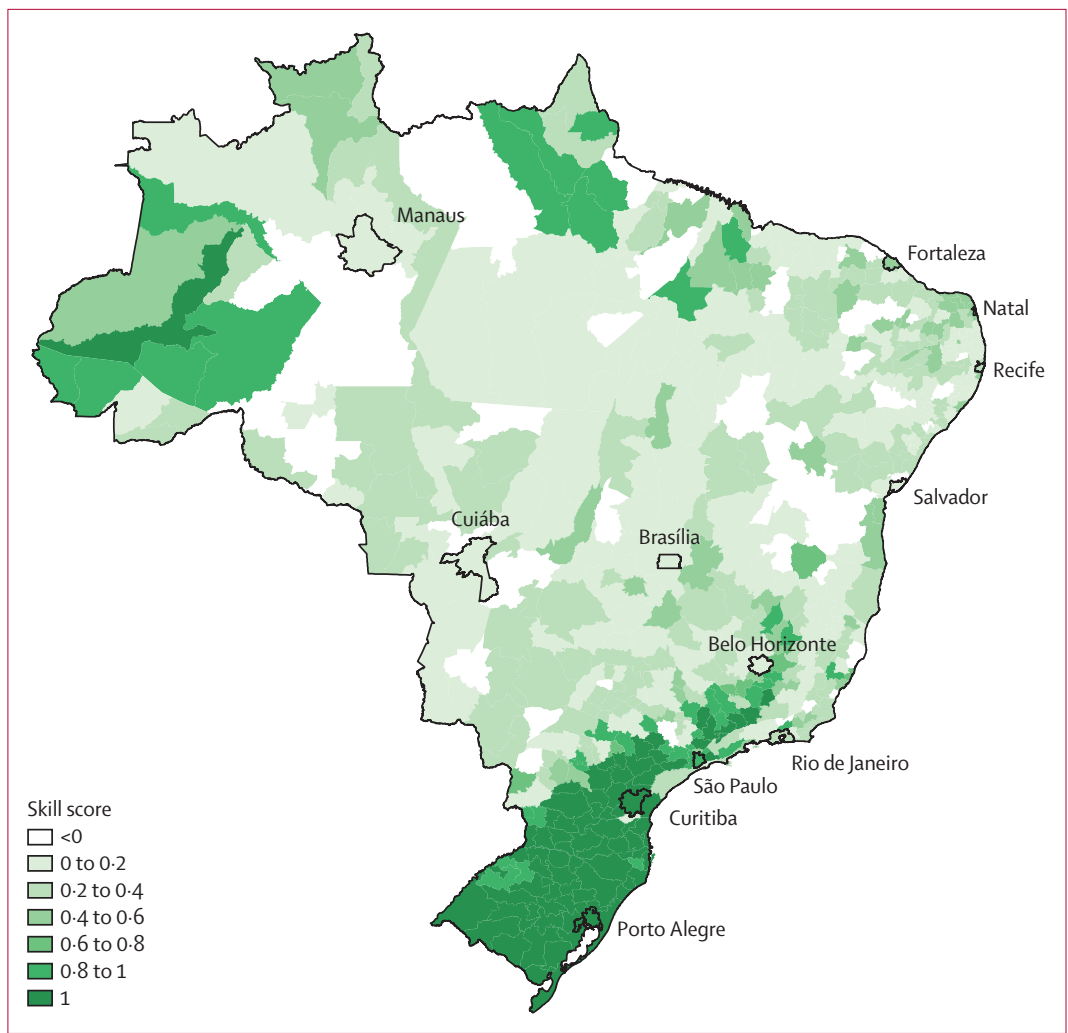

Figure 4: Past performance of forecasting system, June, 2000-13

The rank probability skill score (RPSS) for every microregion based on out-of sample retrospective dengue forecasts June, 2000-13. The RPSS takes the value one for a perfect forecast and zero for the benchmark (reference) forecast. The darker the shade of green, the more skill provided by the forecasting system. Negative values (white) show areas where the model did worse than using the benchmark.
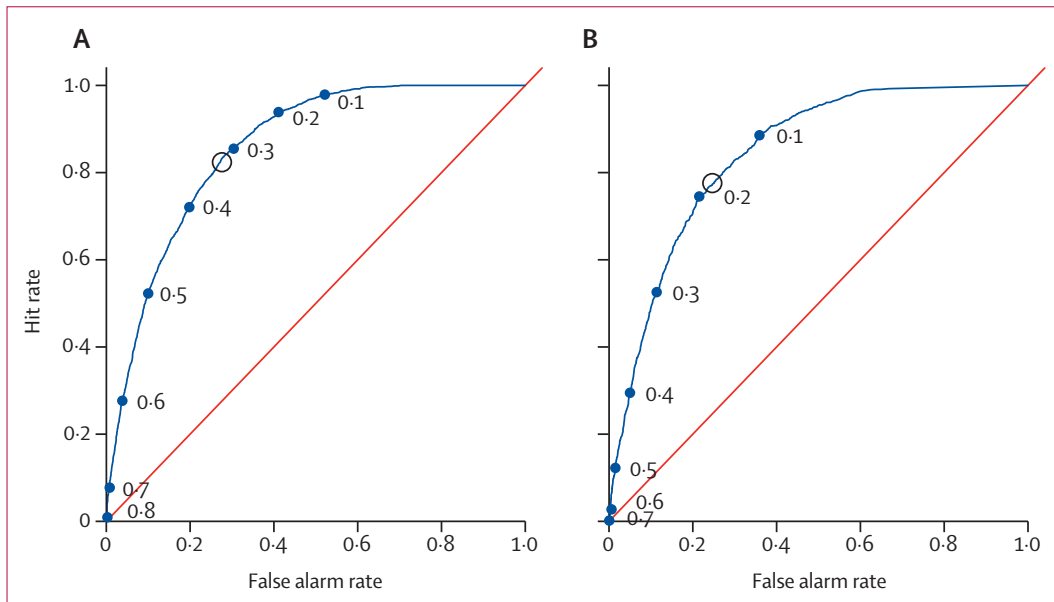

Figure 5: ROC curves to define trigger thresholds

ROC=receiver operating characteristic. $A U C=$ area under the curve. ROC curve for binary event of dengue incidence rates exceeding (A) medium risk threshold of 100 cases and (B) high risk threshold of 300 cases per 100000 inhabitants. Numbers indicate values of probability thresholds along the curve, and circles indicate the position of an optimum ROC cutoff (trigger threshold), defined as the point on the curve closest to the point of perfect discrimination $(0,1)$. The optimum probability trigger threshold for 100 cases is $\sigma=0.32$ (ie, if the probability of low risk is less than 0.68 [1- $\sigma$ ], medium risk warning issued). The optimum trigger threshold for 300 cases is $\rho=0.18$ (ie, if, simultaneously, the probability of high risk category is greater than $0 \cdot 18$, warning upgraded to high risk). As an indicator of the quality of the forecasting system, we calculated the AUC as 0.86 ( $p<0.0001$ ) for (A) and $0.84(p<0.0001)$ for (B). This finding indicates that the forecasting system performs significantly better than randomly guessing (appendix). 
benchmark. The RPSS was positive for all cities where the stadiums are located, indicating some degree of benefit over the benchmark. The level of skill varied widely. Skill was high in cities in the northeast, where the forecast indicated high risk of dengue. Skill was also high in the south region and parts of the Amazon, since the model recognised these areas as typically void of dengue or as sparsely populated. For cities in the interior, skill seemed relatively low. As an indication of the trust a decision maker can place in the forecast for specific microregions, the table lists the skill score (RPSS).

We then looked at the binary classification of a disease exceeding medium and high-risk thresholds (figure 5). We calculated the optimum decision trigger threshold. The optimum probability trigger threshold for medium risk was $\sigma=32 \%$. As such, if the probability of low risk falls under $68 \%$ (ie, $100-\sigma$ ) a medium risk warning should be issued. The optimum trigger threshold for high risk was $\sigma=18 \%$. Therefore, if, concurrently, the probability of high risk exceeds $18 \%$, the warning should be upgraded to high risk.

Using these criteria, we identified dengue risk warnings for every World Cup host city (table). For June, 2014, dengue risk was forecast to be low for the micoregions Brasília, Cuiabá, Curitiba, Porto Alegre, and São Paulo. A medium risk level was assigned to Rio de Janeiro, Belo Horizonte, Salvador, and Manaus. High-risk alerts were triggered for the northeastern cities of Recife, Fortaleza, and Natal, with a probability of exceeding the high-risk epidemic threshold of 19\% for Recife, $46 \%$ for Fortaleza, and $48 \%$ for Natal. For these high-risk areas, particularly Natal, followed by Fortaleza, the forecasting system did well for previous years (2000-13).

\section{Discussion}

Dengue outbreaks during the World Cup are unlikely in cities in south and central Brazil (Brasília, Cuiabá, Curitiba, Porto Alegre, and São Paulo). A medium-risk level is assigned to Rio de Janeiro, Belo Horizonte, Salvador, and Manaus, and the probability of high risk is greatest for the northeastern cities of Natal and Fortaleza, followed by Recife. This forecast suggests that efforts to reduce dengue incidence and severity should be concentrated in these cities.

The impact of mass global travel on infectious diseases transmission has been widely discussed. ${ }^{27}$ However, when the disease is vector-borne, the scenario is not yet well understood. Imposing travel restrictions to affected countries, or creating unnecessary alarm would exclude many parts of the world from hosting international events, such as the World Cup or the Olympics. An approach based on syndromic surveillance and early reporting $^{28}$ is limited for dengue fever, since the transmission is not person-to-person. Therefore, control strategies should be implemented before the arrival of the visitors to Brazil, because of the potentially explosive nature of dengue epidemics. Further, assessments of dengue risk based on expert opinion ${ }^{13}$ are of little use to public health services, compared with objective quantitative risk predictions based on robust, validated methods.

The susceptibility of spectators attending the 2014 World Cup in Brazil will vary depending on the country of origin, the sociodemographic profile of the source population to which the individual belongs, and the duration of visits to each city.

Visitors are not expected to stay in the same city for much longer than 2-3 weeks. Therefore, an epidemic must already be in progress in the population of the host country to allow enough time for large numbers of virus carrying mosquitoes to bite susceptible visitors. The use of a 3 month period for prediction is one of the main advantages of this model, because it will allow sufficient time to initiate massive control of the aedes population, to protect the population of the host country, visitors, and subsequently the visitor population in the country of origin. We do not make any predictions for individual cases, nor do we assess or indicate individual protective behaviour, which is especially complex because of the diurnal habits of aedes. The main contribution of this article is to focus the control of mosquito populations in the cities with an increased probability of exceeding epidemic dengue risk levels.

During the austral summer 2013-14, the number of dengue cases decreased across Brazil by about $80 \%$, compared with the previous year. This decrease was mainly due to two factors: the occurrence of an atypical summer, with exceptionally hot and dry conditions in the south and southeast, and the occurrence of a large dengue epidemic in 2013. The atypical summer resulted in a decrease in mosquito infestation in most cities, and the epidemic in a depletion of the susceptible population. The accumulation of the susceptible population is a necessary condition to trigger an epidemic. ${ }^{29}$ These factors align with the very low risk of dengue outbreaks in Porto Alegre, Curitiba, and São Paulo during the forthcoming World Cup. The Amazon experienced more rainfall than usual during summer, providing suitable conditions for dengue transmission. This heavy rainfall, along with the forecast of positive precipitation anomalies in March, April, and May, increases the probability of dengue risk in June exceeding medium levels in, for example, Manaus. The probability of dengue outbreaks is greatest for Natal and Fortaleza, followed by Recife, which aligns with the forecast of positive precipitation anomalies for the northeastern region of Brazil. This region also showed good skill in predicting past dengue risk.

Austral autumn (March, April, and May) is the main precipitation season for the northeastern region of Brazil. This precipitation is influenced by the southward movement of the intertropical convergence zone 
(ITCZ). ${ }^{30,31}$ When the ITCZ migrates to the south, this region experiences intense precipitation events. When this movement does not occur, severe drought conditions are noted. The migration of the ITCZ over northeastern Brazil is connected to sea surface temperature conditions both in the tropical Pacific and Atlantic oceans. ${ }^{32}$ Generally, precipitation increases in the northeastern region of Brazil are associated with colder than normal conditions in the tropical Pacific (eg, La Niña events), colder than normal sea surface temperature conditions in the tropical north Atlantic, ${ }^{33}$ and warmer than normal sea surface temperature conditions in the tropical south Atlantic. ${ }^{34}$ The reverse is noted during El Niño events. The dynamic models used to produce the EUROBRISA forecasts are generally able to reproduce such precipitation patterns..$^{20}$

This early warning system is based on a spatiotemporal Bayesian hierarchical model framework driven by climate and non-climate information. Inclusion of random effects accounts for unobserved confounding factors (eg, presence of mosquito predators or complex serotype interactions) and spatial and temporal dependency structures within different ecological zones. Probabilistic forecasts allow prediction uncertainty to be quantified and translated into geographically specific dengue early warnings. The analysis benefited from a novel procedure for visualising ternary probability forecasts that uses colour saturation to differentiate between different levels of forecast certainty, rather than a single shade to represent a range of forecast outcomes. ${ }^{25}$

We did a thorough assessment of the past performance of the dengue early warning system by verifying probabilistic predictions against out-of-sample data. Providing an honest assessment of the forecasting system is a vital component in the communication of forecast uncertainty to decision makers. The limitations of the forecasting system must be taken into account when allocating scarce resources.

Despite limitations, the ability to provide early warnings of dengue epidemics at the microregion level, 3 months in advance, is valuable for public health decision making and intervention. Based on early warnings, control strategies could move from dealing with clinically severe dengue and death prevention to intensively combating mosquito populations, particularly in those cities with a greater chance of high dengue risk (Natal, Fortaleza, and Recife). This is not feasible on a day-to-day basis, but reasonable under an epidemic alert. Year-round larval control can be counterproductive, exacerbating epidemics in later years because of evolution of insecticide resistance and loss of herd immunity. ${ }^{35}$ This dengue early warning system, driven by seasonal climate information could greatly aid the management of scarce resources throughout the year.

As well as informing local authorities as to the potential dengue risk during the event, these early warnings provide
Panel: Research in context

\section{Systematic review}

We searched PubMed on May 7, 2014, with the terms "climate", "dengue", "early warning", "model". A recent systematic review highlighted several other studies that model the impact of climate on dengue transmission. ${ }^{10}$ However, none of these studies have incorporated real-time seasonal climate forecasts to make dengue predictions. We propose a probabilistic dengue model, using the best data and methods available. ${ }^{18-20,25}$ This more realistic climate-driven dengue early warning can inform public health officials, without creating unnecessary alarm.

\section{Interpretation}

The dengue risk warnings presented here have utility and significance to public health decision makers, well beyond expert assessment based on historical averages. This dengue probabilistic forecast is based on a sophisticated spatiotemporal model framework, driven by real-time seasonal climate forecasts. This study contributes an unprecedented prototype of a climate-informed public health support system. The forecast provides an early example of the interdisciplinary research, development and application envisaged for the Global Framework for Climate Services (GFCS) ${ }^{36}$ developing under the UN system and spearheaded by the World Meteorological Organisation. The GFCS aims to mainstream climate information into decision making at all levels in society, with health selected as one of four priority sectors.

football fans with more information than currently available via press reports regarding the potential dengue risk situation in Brazil during the World Cup. The successful implementation of seasonal climate forecasts in disease early warning systems depends on close collaboration between public health specialists, climate scientists, and mathematical modellers. To our knowledge, this is the first example of a climate service for public health, ahead of a major global event (panel).

\section{Contributors}

RL was responsible for the study design, data collation, model development, data analysis, and wrote the manuscript. CB proposed the study, was responsible for collating and cleaning the dengue, cartographic, and socio-economic data, interpreting results, and wrote parts of the introduction and discussion. CASC produced the real-time and past climate forecasts for the study, wrote the climate forecast methods, contributed to the description of the skill and dynamics of climate prediction in Brazil, and edited the report. TCB contributed to model development and related statistical or computational issues. GEC helped with the use of the latest surveillance dengue data to make the real-time forecast and provided a health policy interpretation of the forecast. RG provided input and guidance about the climate forecasts, expert advice about the relevance of the study for the GFCS, and edited the report. TJ provided technical and computational assistance for the visualisation of the probabilistic forecasts and reviewed the methods. WMR cleaned and collated the dengue data, including the most up-todate cases from the Ministry of Health, and helped interpret the results. MSC contributed to model development and presentation of results, provided health-epidemiological interpretation of the data and wrote parts of the introduction and discussion. DBS provided statistical advice on how to issue and present the probability forecast and provided editorial advice. XR contributed to literature search, data interpretation and helped write the report. All authors discussed the results, reviewed, and approved the final report.

Declaration of interests

We declare no competing interests.

Acknowledgments

The research leading to these results has received funding from the DENFREE project (grant agreement $n^{\circ} 282378$ ), EUPORIAS project (grant 
agreement $n^{\circ}$ 308291) and SPECS project (grant agreement $n^{\circ} 308378$ ) funded by the European Commission's Seventh Framework Research Programme. CASC was supported by Conselho Nacional de Desenvolvimento Científico e Tecnológico (CNPq) process 306863/2013-8. MSC received funding from Conselho Nacional de Desenvolvimento Científico e Tecnológico $(\mathrm{CNPq})$ and Fundação de Amparo à Pesquisa do Estado do Rio de Janeiro (FAPERJ). The dynamical ensemble forecast data were kindly provided by ECMWF as part of the EUROBRISA licence agreement. We thank Josep Manel Andrés and Albert Jornet Puig for facilitating computational resources and providing technical support. We also thank the five anonymous reviewers who helped strengthen the arguments of this Article.

References

1 Guzman MG, Halstead SB, Artsob H, et al. Dengue: a continuing global threat. Nat Rev Microbiol 2010; 8: S7-S16.

2 Whitehorn J, Farrar J. Dengue. Br Med Bull 2010; 95: 161-173.

3 Gubler DJ. The economic burden of dengue. Am J Trop Med Hyg 2012; 86: 743.

4 Hsieh YH, Chen CWS. Turning points, reproduction number, and impact of climatological events for multi-wave dengue outbreaks. Trop Med Int Health 2009; 14: 628-638.

5 Lana RM, Carneiro TG, Honório NA, Codeço CT. (2014). Seasonal and nonseasonal dynamics of Aedes aegypti in Rio de Janeiro, Brazil: Fitting mathematical models to trap data. Acta Trop 2014; 129: 2532.

6 Johansson MA, Cummings DAT, Glass GE. Multi-year variability and dengue-El Niño Southern Oscillation, weather, and dengue incidence in Puerto Rico, Mexico, and Thailand: a longitudinal data analysis. PLoS Med 2009; 6: 11.

7 Chowell G, Cazelles G, Broutin H, Munayco CV. The influence of geographic and climate factors on the timing of dengue epidemics in Perú, 1994-2008. BMC Infect Dis 2011; 11: 164

8 Banu S, Hu W, Hurst C, Tong S. Dengue transmission in the Asia-Pacific region: impact of climate change and socio-environmental factors. Trop Med Int Health 2011; 16: 598-607.

9 Scott TW, Morrison AC. Vector dynamics and transmission of dengue virus: implications for dengue surveillance and prevention strategies. Curr Top Microbiol Immunol 2010; 338: 115-28.

10 Naish S, Dale P, Mackenzie JS, McBride J, Mengersen K, Tong S. Climate change and dengue: a critical and systematic review of quantitative modelling approaches. BMC Infect Dis 2014; 14: 167

11 Teixeira MG, Costa MCN, Barreto F, Barreto ML. Dengue: twentyfive years since reemergence in Brazil. Cad Saúde Pública 2009; 25: 7-18

122010 FIFA World Cup South Africa. http://www.fifa.com/ tournaments/archive/worldcup/southafrica2010/index.html (accessed May 5, 2014)

13 Hay S. Football fever could be a dose of dengue. Nature 2013 503: 439.

14 Massad E, Wilder-Smith A. Risk estimates of dengue in travellers to dengue endemic areas using mathematical models. J Travel Med 2009; 16: 191-93.

15 Ministry of Health. National guidelines for prevention and control of dengue epidemics. Brasilia, 2009. http://www.combateadengue. pr.gov.br/arquivos/File/profissionais/diretrizes_dengue.pdf (accessed April 6, 2014)

16 Marra AR, de Matos GF, Janeri, RD, Machado PS, Schvartsman C, dos Santos OF. Managing patients with dengue fever during an epidemic: the importance of a hydration tent and of a multidisciplinary approach. BMC Res Notes 2011; 4: 335.

17 Resende MCD, Silva IM, Ellis BR, Eiras AE. A comparison of larval, ovitrap and MosquiTRAP surveillance for Aedes (Stegomyia) aegypti. Mem Inst Oswaldo Cruz 2013; 108: 1024-30.
18 Lowe, R. Bailey TC, Stephenson DB, et al. Spatiotemporal modelling of climate-sensitive disease risk: towards an early warning system for dengue in Brazil. Comput Geosci 2011 37: $371-81$.

19 Lowe R, Bailey TC, Stephenson DB, et al. The development of an early warning system for climate-sensitive disease risk with a focus on dengue epidemics in Southeast Brazil. Stat Med 2013 32: 864-83.

20 Coelho CAS, Stephenson DB, Balmaseda M, Doblas-Reyes FJ, van Oldenborgh GJ. Toward an integrated seasonal forecasting system for South America. J Climate 2006; 19: 3704-21.

21 Fan Y, Van den Dool H. A global monthly land surface air temperature analysis for 1948-present. J Geophys Res 2008; 113: D1.

22 Adler RF, Huffman GJ, Chang A, et al. The version-2 Global Precipitation Climatology Project (GPCP) monthly precipitation analysis (1979-present). J Hydrometeorol 2003; 4: 1147-67.

23 Stephenson DB, Coelho CAS, Doblas-Reyes FJ, Balmaseda M. Forecast assimilation: a unified framework for the combination of multi-model weather and climate predictions. Tellus A 2005; 57: 253-64.

24 Gelman A, Meng XL, Stern H. Posterior predictive assessment of model fitness via realized discrepancies. Stat Sinica 1996; 6: 733-59.

25 Jupp TE, Lowe R, Coelho CAS, Stephenson DB. On the visualization, verification and recalibration of ternary probabilistic forecasts. Phil Trans R Soc A 2012; 370: 1100-20.

26 Jolliffe IT, Stephenson DB. Forecast verification: a practitioner's guide in atmospheric science. 2nd Edn. Chichester: Wiley, 2011.

27 Shi P, Keskinocak P, Swann JL, Lee BY. The impact of mas gatherings and holiday traveling on the course of an influenza pandemic: a computational model. BMC Public Health 2010; 10: 778.

28 Severi E, Heinsbroek E., Watson C, et al. Infectious disease surveillance for the London 2012 Olympic and Paralympic Games. Euro Surveill 2012; 17: 1-8.

29 Chowell G, Diaz-Dueñas P, Miller JC, et al. Estimation of the reproduction number of dengue fever from spatial epidemic data. Math Biosci 2007; 208: 571-89.

30 Giannini A, Chiang JCH, Cane M, Kushnir Y, Seager R. The ENSO teleconnection to the tropical Atlantic Ocean: contributions of the remote and local SSTs to rainfall variability in the tropical Americas. J Climate 2001; 14: 4530-44.

31 Ma HY, Ji X, Neelin JD, Mechoso CR. Mechanisms for precipitation variability of the eastern Brazil/SACZ convective margin. $J$ Climate 2011; 24: 3445-3456.

32 Uvo CRB, Repelli CA, Zebiak S, Kushnir Y. The relationships between tropical Pacific and Atlantic SST and northeast Brazil monthly precipitation. J Climate 1998; 11: 551-62.

33 Rodríguez-Fonseca B, Polo I, García-Serrano J, et al. Are Atlantic Niños enhancing Pacific ENSO events in recent decades? Geophys Res Let 2009; 36: L20705.

34 Folland CK, Colman AW, Rowell DP, Davey MK1. Predictability of northeast Brazil rainfall and real-time forecast skill, 1987-98. J Climate 2001; 14: 1937-58.

35 Luz PM, Vanni T, Medlock J, Paltiel AD, Galvani, AP. Dengue vector control strategies in an urban setting: an economic modelling assessment. Lancet 2011; 377: 1673-80.

36 Hewitt C, Mason S, Walland D. The global framework for climate services. Nature Clim Change 2012; 2: 831-32. 